\title{
CHALMERS
}

Progress Report

\section{LARGE EDDY SIMULATION OF A NATURAL CONVECTION BOUNDARY LAYER ON A VERTICAL CYLINDER}

Darioush G. Barhaghi and Lars Davidson

Department of Thermo and Fluid Dynamics Chalmers University of TeChNology Göteborg, Sweden, April 2003 


\title{
LARGE EDDY SIMULATION OF A NATURAL CONVECTION BOUNDARY LAYER ON A VERTICAL CYLINDER
}

\author{
Darioush G. Barhaghi and Lars Davidson \\ Dept. of Thermo and Fluid Dynamics \\ Chalmers University of Technology \\ SE-412 96 Göteborg, Sweden
}




\section{Contents}

$\begin{array}{ll}\text { Abstract } & 4\end{array}$

Nomenclature $\quad 5$

$\begin{array}{lll}1 & \text { Introduction } & 7\end{array}$

$\begin{array}{lll}2 & \text { Governing equations } & 7\end{array}$

3 Numerical method $\quad 11$

3.1 Boundary conditions . . . . . . . . . . . 11

3.2 Numerical oscillations . . . . . . . . . . . . . . 11

4 Results 12

5 Near future work $\quad 12$ 


\begin{abstract}
Large Eddy Simulation (LES) is used to study the natural convection boundary layer and its behavior on a vertical heated cylinder of the flow inside a vertical shell and tube. This is a progress report on the work carried out problems regarding implementation of different boundary conditions and different numerical schemes are discussed.
\end{abstract}




\section{Nomenclature}

\section{Latin Symbols}

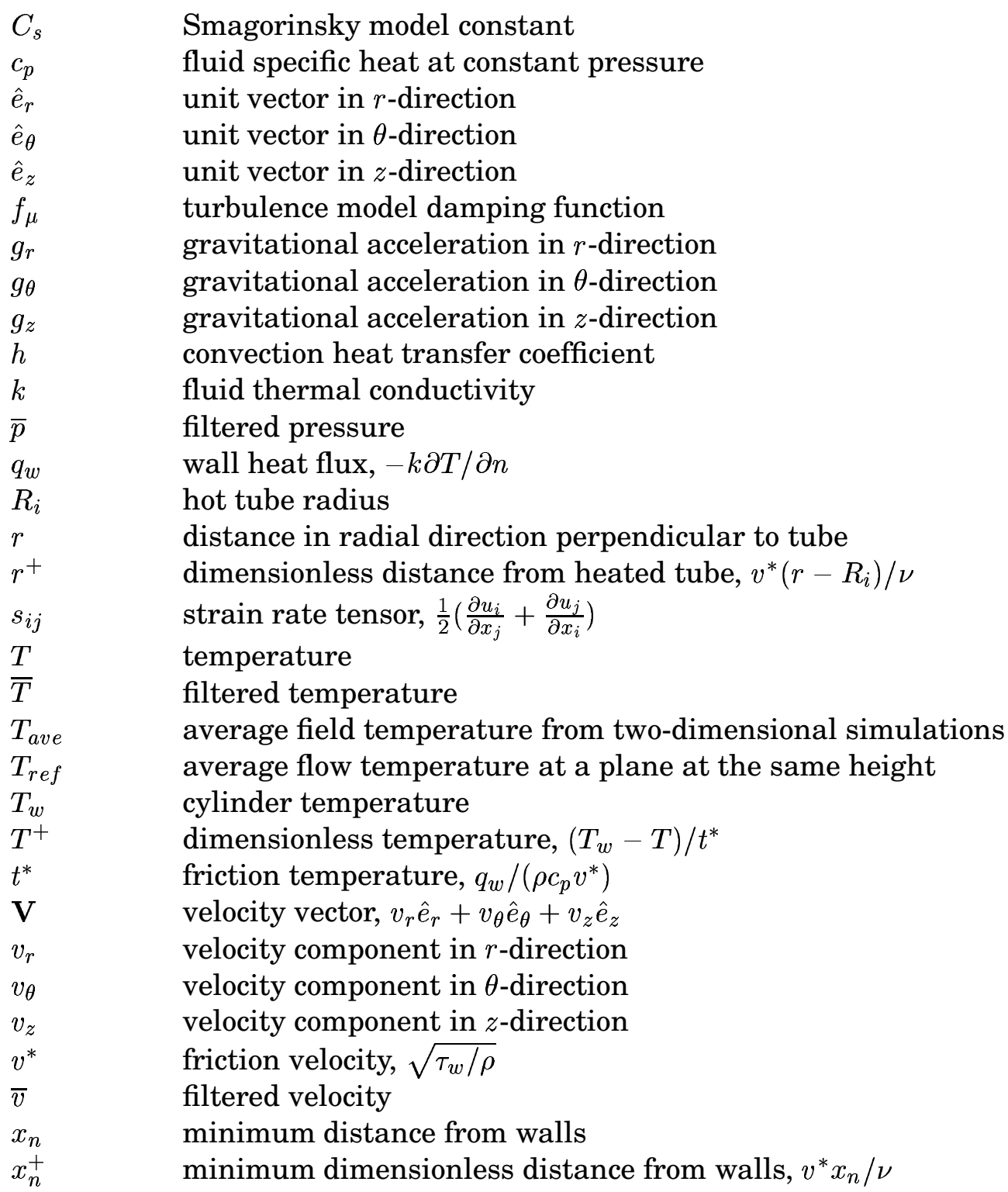




\section{Greek Symbols}

$\begin{array}{ll}\alpha & \text { thermal diffusivity, } k /\left(\rho c_{p}\right) \\ \beta & \text { coefficient of expansion, } 2 /\left(T_{w}[K]+T_{\text {ave }}[K]\right) \\ \delta V_{I J K} & \text { local grid size, } r_{I} \delta \theta_{K} \delta y_{J} \delta r_{I} \\ \Delta & \text { filter width, }\left(\delta V_{I J K}\right)^{\frac{1}{3}} \\ \mu & \text { fluid dynamic viscosity } \\ \nabla & \text { gradient vector, } \frac{\partial}{\partial r} \hat{e}_{r}+\frac{\partial}{r \partial \theta} \hat{e}_{\theta}+\frac{\partial}{\partial z} \hat{e}_{z} \\ \nu_{e f f} & \text { effective kinematic viscosity } \\ \nu_{s g s} & \text { sub-grid scale eddy viscosity } \\ \rho & \text { fluid density } \\ \tau_{i j} & \text { stress tensor } \\ \tau_{w} & \text { wall shear stress, } \mu \partial v / \partial x_{n} \\ \theta & \text { azimuthal angle in cylindrical coordinate system }\end{array}$




\section{Introduction}

As natural convection heat transfer is an important phenomena which is dominant in a number of important and interesting flows, it is of large interest to study a generic natural convection boundary layer. With the advent of stronger computers with a larger capacity, Large Eddy Simulation (hereafter LES) has opened an interesting tool to study this flow.

The geometry for which current calculations have been carried out is based on an experimental shell and tube which is designed to study the natural convection heat transfer from a vertical cylinder and is shown in figure 1. The simplified geometry is based on the real experimental rig shown in figure 2. The simplified configuration was evaluated and tested using RANS by Barhaghi et al. (2003).

As LES computations require considerable time for achieving a fully developed flow, the initial boundary conditions are taken from a twodimensional RANS calculations (Barhaghi et al., 2003).

\section{Governing equations}

The filtered Navier-Stokes equations in cylindrical coordinates read

Continuity:

$\frac{1}{r} \frac{\partial}{\partial r}\left(r \bar{v}_{r}\right)+\frac{1}{r} \frac{\partial}{\partial \theta}\left(\bar{v}_{\theta}\right)+\frac{\partial}{\partial z}\left(\bar{v}_{z}\right)=0$

Convective time derivative:

$\mathbf{V} \cdot \nabla=\bar{v}_{r} \frac{\partial}{\partial r}+\frac{1}{r} \bar{v}_{\theta} \frac{\partial}{\partial \theta}+\bar{v}_{z} \frac{\partial}{\partial z}$

Laplacian operator:

$\nabla^{2}=\frac{1}{r} \frac{\partial}{\partial r}\left(r \frac{\partial}{\partial r}\right)+\frac{1}{r^{2}} \frac{\partial^{2}}{\partial \theta^{2}}+\frac{\partial^{2}}{\partial z^{2}}$

The $r$-momentum equation:

$$
\begin{aligned}
\frac{\partial \bar{v}_{r}}{\partial t}+(\mathbf{V} \cdot \nabla) \bar{v}_{r}-\frac{1}{r} \bar{v}_{\theta}^{2} & =-\frac{1}{\rho} \frac{\partial p}{\partial r}+g_{r}+\frac{1}{r} \frac{\partial}{\partial r}\left(r \nu_{e f f, v} \frac{\partial \bar{v}_{r}}{\partial r}\right)+ \\
\frac{1}{r^{2}} \frac{\partial}{\partial \theta}\left(\nu_{e f f, v} \frac{\partial \bar{v}_{r}}{\partial \theta}\right) & +\frac{\partial}{\partial z}\left(\nu_{e f f, v} \frac{\partial \bar{v}_{r}}{\partial z}\right)-\nu_{e f f, v} \frac{\bar{v}_{r}}{r^{2}}-\frac{2}{r^{2}} \nu_{e f f, v} \frac{\partial \bar{v}_{\theta}}{\partial \theta}
\end{aligned}
$$




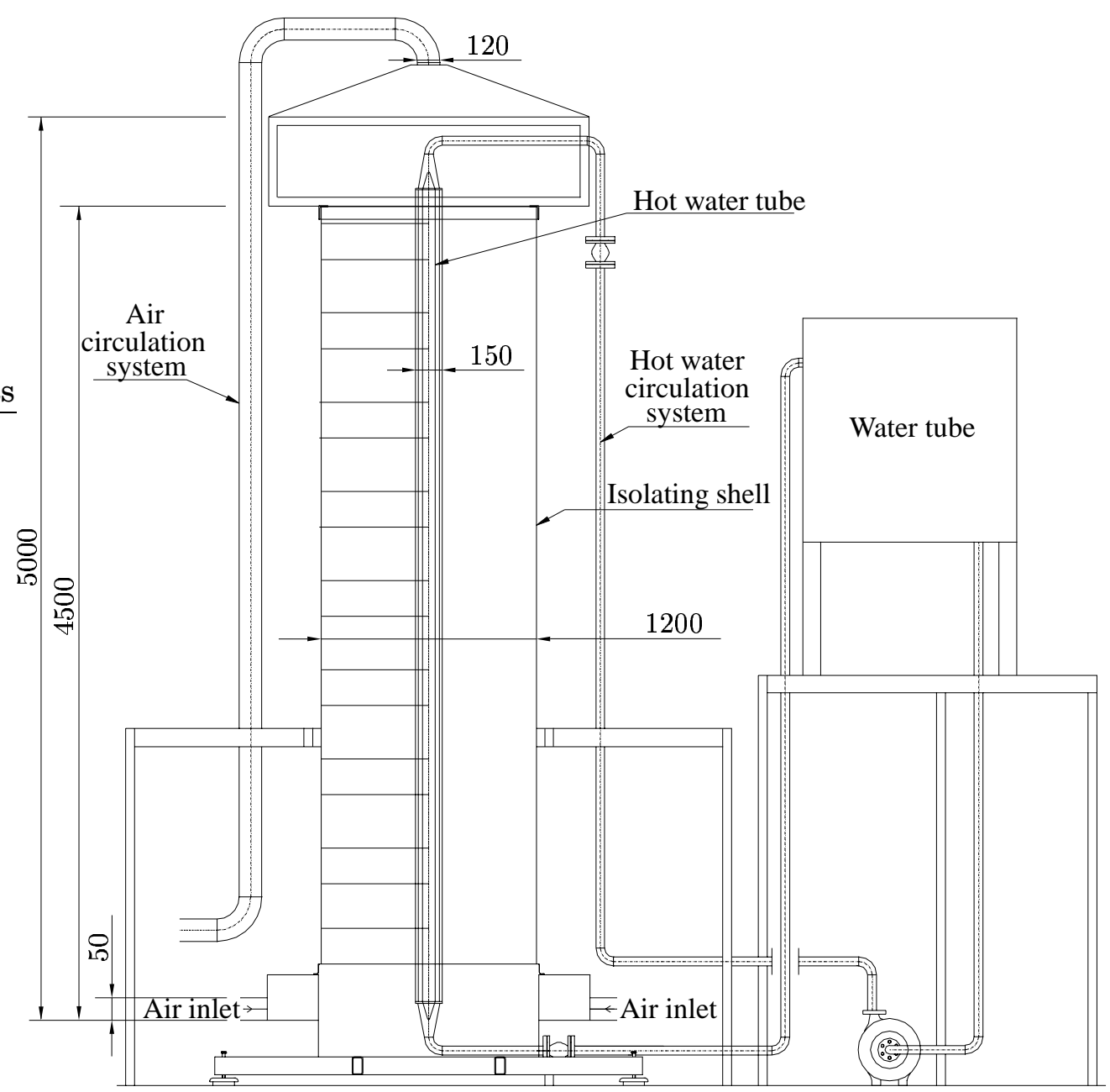

Figure 1: Experimental rig

The $\theta$-momentum equation:

$$
\begin{aligned}
\frac{\partial \bar{v}_{\theta}}{\partial t}+ & (\mathbf{V} \cdot \nabla) \bar{v}_{\theta}+\frac{1}{r} \bar{v}_{r} \bar{v}_{\theta}=-\frac{1}{\rho r} \frac{\partial p}{\partial \theta}+g_{\theta}+\frac{1}{r} \frac{\partial}{\partial r}\left(r \nu_{e f f, v} \frac{\partial \bar{v}_{\theta}}{\partial r}\right)+ \\
& \frac{1}{r^{2}} \frac{\partial}{\partial \theta}\left(\nu_{e f f, v} \frac{\partial \bar{v}_{\theta}}{\partial \theta}\right)+\frac{\partial}{\partial z}\left(\nu_{e f f, v} \frac{\partial \bar{v}_{\theta}}{\partial z}\right)-\nu_{e f f, v} \frac{\bar{v}_{\theta}}{r^{2}}+\frac{2}{r^{2}} \nu_{e f f, v} \frac{\partial \bar{v}_{r}}{\partial \theta}
\end{aligned}
$$




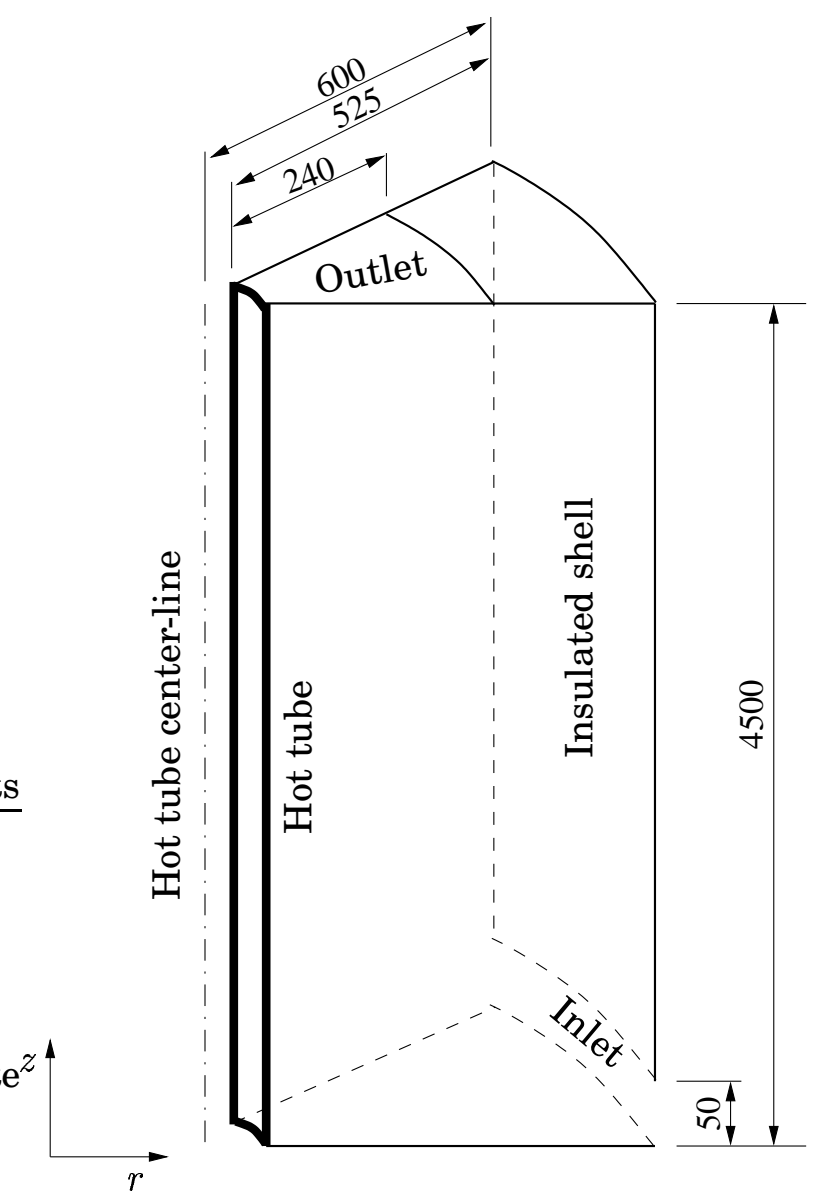

Figure 2: Computational geometry

The $z$-momentum equation:

$$
\begin{aligned}
\frac{\partial \bar{v}_{z}}{\partial t}+ & (\mathbf{V} \cdot \nabla) \bar{v}_{z}=-\frac{1}{\rho} \frac{\partial p}{\partial z}+g_{z} \beta\left(\bar{T}-T_{r e f}\right)+ \\
& \frac{1}{r} \frac{\partial}{\partial r}\left(r \nu_{e f f, v} \frac{\partial \bar{v}_{z}}{\partial r}\right)+\frac{1}{r^{2}} \frac{\partial}{\partial \theta}\left(\nu_{e f f, v} \frac{\partial \bar{v}_{z}}{\partial \theta}\right)+\frac{\partial}{\partial z}\left(\nu_{e f f, v} \frac{\partial \bar{v}_{z}}{\partial z}\right)
\end{aligned}
$$


The energy equation:

$$
\begin{aligned}
& \frac{\partial \bar{T}}{\partial t}+(\mathbf{V} \cdot \nabla) \bar{T}= \\
& \quad \frac{1}{r} \frac{\partial}{\partial r}\left(r \nu_{\text {eff }, T} \frac{\partial \bar{T}}{\partial r}\right)+\frac{1}{r^{2}} \frac{\partial}{\partial \theta}\left(\nu_{\text {eff }, T} \frac{\partial \bar{T}}{\partial \theta}\right)+\frac{\partial}{\partial z}\left(\nu_{\text {eff }, T} \frac{\partial \bar{T}}{\partial z}\right)
\end{aligned}
$$

in which:

$$
\begin{aligned}
& p=\bar{p}+\frac{1}{3} \rho \tau_{k k} \\
& \nu_{e f f, v}=\nu+\nu_{s g s} \\
& \nu_{e f f, T}=\frac{\nu}{P r}+\frac{\nu_{s g s}}{\operatorname{Pr}_{t}} \\
& \nu_{s g s}=\left(C_{S} \Delta\right)^{2} f_{\mu} \sqrt{2 \bar{s}_{i j} \bar{s}_{i j}} \\
& 2 \bar{s}_{i j} \bar{s}_{i j}=2\left[\left(\frac{\partial \bar{v}_{r}}{\partial r}\right)^{2}+\left(\frac{1}{r} \frac{\partial \bar{v}_{\theta}}{\partial \theta}+\frac{\bar{v}_{r}}{r}\right)^{2}+\left(\frac{\partial \bar{v}_{z}}{\partial z}\right)^{2}\right]+\left[\left(\frac{\partial \bar{v}_{r}}{\partial z}+\frac{\partial \bar{v}_{z}}{\partial r}\right)^{2}\right. \\
& \left.+\left(\frac{1}{r} \frac{\partial \bar{v}_{r}}{\partial \theta}+\frac{\partial \bar{v}_{\theta}}{\partial r}-\frac{\bar{v}_{\theta}}{r}\right)^{2}+\left(\frac{\partial \bar{v}_{\theta}}{\partial z}+\frac{1}{r} \frac{\partial \bar{v}_{z}}{\partial \theta}\right)^{2}\right] \\
& f_{\mu}=1-\exp \left(-\frac{x_{n}^{+}}{25}\right) \\
& C_{S}=0.1, \quad P r_{t}=0.4, \quad \operatorname{Pr}=0.7
\end{aligned}
$$

In the above equations, the turbulent diffusive cross terms arising from $\frac{\partial}{\partial x_{j}}\left(\nu_{\text {eff }} \frac{\partial \bar{v}_{j}}{\partial x_{i}}\right)$ in which $\bar{v}_{j}$ stands for filtered velocity vector, are neglected. 


\section{Numerical method}

An incompressible, finite volume code is used (Davidson \& Peng, 2003). For space discretization, central differencing is used for all terms. The Crank-Nicolson scheme is used for time discretization. The numerical procedure is based on an implicit, fractional step technique with a multi-grid pressure Poisson solver and a non-staggered grid arrangement (Emvin, 1997).

\subsection{Boundary conditions}

For the hot tube wall the temperature is set to $80^{\circ} \mathrm{C}$ and Neumann temperature boundary condition are used for the other walls. The inlet temperature is $T=25^{\circ} \mathrm{C}$. This yields a maximum Grashof number of $4.5 \cdot 10^{11}$ and a Rayleigh number of $3.2 \cdot 10^{11}$. For the inlet, the Direct Numerical Simulation (DNS) results of a turbulent channel flow with an average velocity of $0.6 \mathrm{~m} / \mathrm{s}$ is imposed. At the outlet, convective boundary condition for velocities and Neumann boundary condition for temperature is used. Periodic boundary conditions are used in the circumferential direction. The spanwise extent (circumferential direction) is set to $18^{\circ}$.

\subsection{Numerical oscillations}

The central differencing scheme was initially used. It is important to use a discretization scheme with low/no numerical dissipation. However, this approach can give rise to unphysical fluctuations due to unboundedness of the central differencing scheme.

Severe problems of unphysical fluctuations have been encountered. Temperatures below $0^{\circ} C$ (!) were detected (recall that the lowest temperature should be $25^{\circ} \mathrm{C}$ ). The critical area was found to be the inlet region where laminar inlet boundary conditions were used. Two main approaches have been used to solve this problem.

1. Local use (both in space and time) of a bounded scheme (the van Leer scheme) for the temperature equation. The criteria for switching to the van Leer scheme is when $T<25^{\circ} \mathrm{C}$ or $T>80^{\circ} \mathrm{C}$. At 
nodes for which $25^{\circ} \mathrm{C} \leq T \leq 80^{\circ} \mathrm{C}$ central differencing is used. This approach works fine.

2. Use of turbulent instantaneous inlet boundary conditions. The turbulence is generated by carrying out a DNS-simulation of fully developed plane channel flow at $R e_{\tau}=500$. In the DNS-simulation instantaneous planes with $U, V, W$ and $T$ were stored on disk and they are used for prescribing instantaneous inlet profiles in the shell and tube simulation. With this approach we can use the nondissipative central differencing scheme everywhere.

\section{Results}

Some preliminary computations have been carried out. A grid with $400 \times$ $96 \times 32$ (vertical, horizontal, circumferential) cells is used. In figure 3 , velocity and temperature at different Grashof numbers $G r_{x}$ are shown.

Although the results are very preliminary, it can be noted that contrary to usual forced convection boundary layers, no logarithmic region is found in the natural convection boundary layer along the hot inner wall. This is in agreement with the theory in George \& Capp (1979). The maximum velocity region is located at $y^{+} \simeq 30$. This is in agreement with experimental findings in Tsuji \& Nagano (1988). In Tsuji \& Nagano (1988) a linear region in the mean temperature profile is found, but not for the mean velocity profile. In the present preliminary simulations the linear region is very small both for temperature and velocity (inside $y^{+} \simeq 1$ ). However, for the mixed convection in the infinite channel Davidson et al. (2003) both velocity and temperature profiles exhibit a linear region very similar to a forced convection boundary layer.

Figures 4 and 5 show the resolved Reynolds stresses at different heights.

\section{Near future work}

The computations will be carried out using many different grids. We are especially concerned about the spanwise extent $\left(z_{\max }\right)$ and the spanwise resolution $\left(\Delta z^{+}\right)$. Also the effect of the streamwise resolution and the wall-normal resolution will be investigated. We will also study the effect 


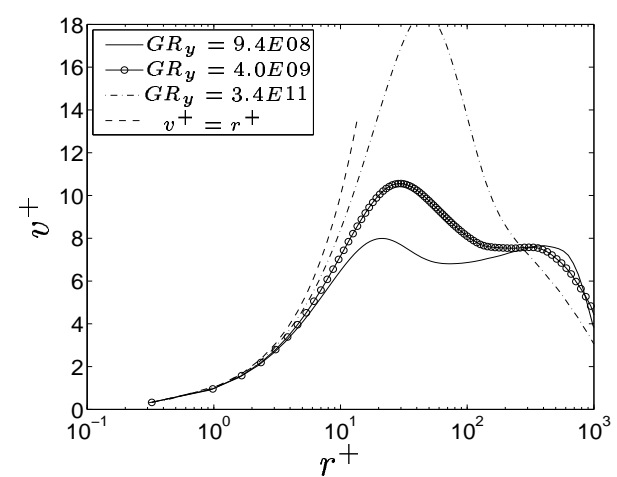

(a) Velocity profile

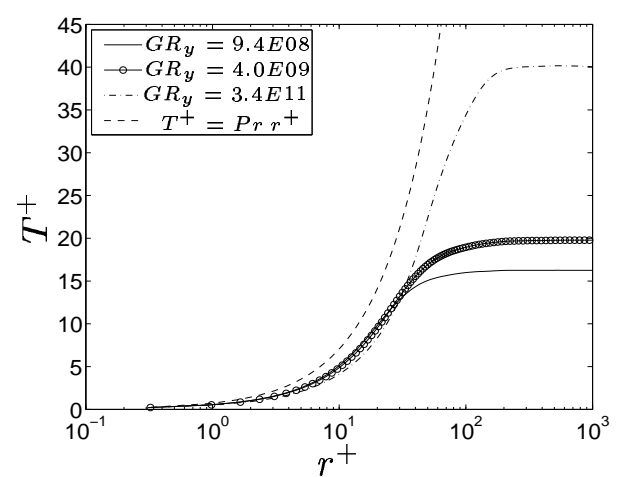

(b) Temperature profile

Figure 3: Velocity and temperature profiles

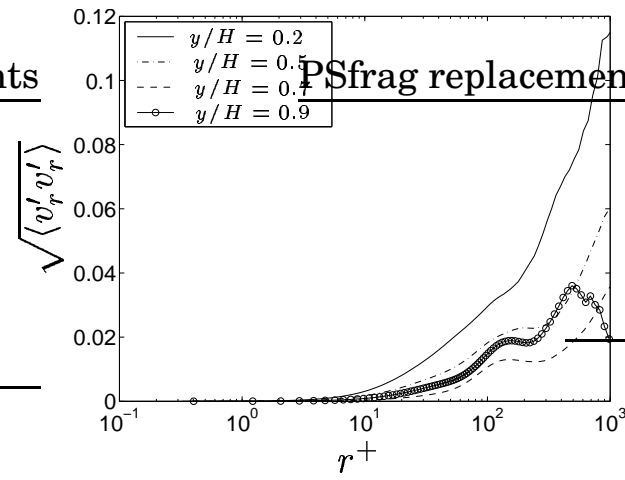

(a)

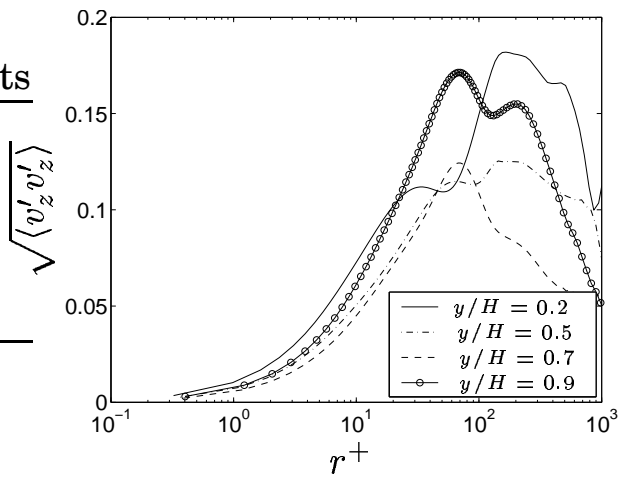

(b)

Figure 4: Reynolds stresses

of the SGS model. It is expected that this should have no significant effect. When this comprehensive grid study has been carried out, the results will be analyzed in detail, both instantaneously and in the mean. 


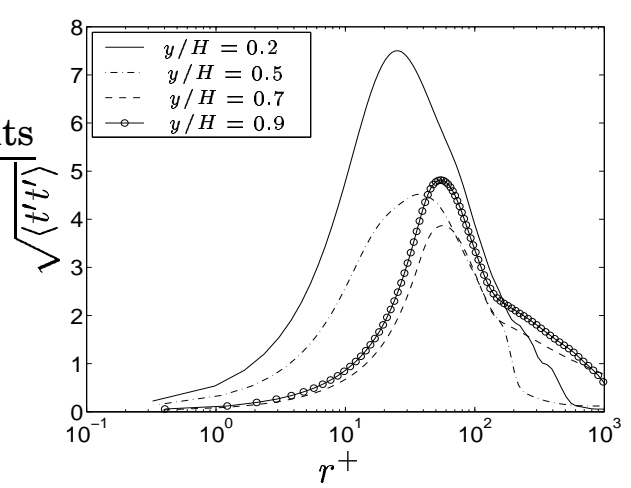

Figure 5: Reynolds stress

\section{Acknowledgments}

This project was financed by the Swedish Research Council, project number 260-1999-354

\section{References}

BARHAGHi, D., DAvidson, L. \& KARLsson, R. 2003 Natural convection heat transfer in a vertical shell and tube. Report 03/01 (can be downloaded from www.tfd. chalmers/ lada/allpaper.html). Dept. of Thermo and Fluid Dynamics, Chalmers University of Technology, Göteborg, Sweden.

Davidson, L., ČUturić, D. \& PENG, S.-H. 2003 DNS in a plane vertical channel with and without buoyancy (to be presented). In 4th Int. Symp. on Turbulence Heat and Mass Transfer. Antalya, Turkey (can be downloaded from www.tfd. chalmers/ lada/allpaper.html).

DAVIDSON, L. \& PENG, S.-H. 2003 Hybrid LES-RANS: A one-equation SGS model combined with a $k-\omega$ model for predicting recirculating flows (to appear). International Journal for Numerical Methods in Fluids .

Emvin, P. 1997 The full multigrid method applied to turbulent flow in ventilated enclosures using structured and unstructured grids. PhD 
thesis, Dept. of Thermo and Fluid Dynamics, Chalmers University of Technology, Göteborg.

GEORGE, W. \& CAPP, S. 1979 A theory for natural convection turbulent boundary layers next to heated vertical surfaces. International Journal of Heat and Mass Transfer 22, 813-826.

TsUJI, T. \& NAGANO, Y. 1988 Characteristics of a turbulent natural convection boundary layer along a vertical flat plate. International Journal of Heat and Mass Transfer 31 (8), 1723-1734. 